\title{
Islet Cell Transplantation for Patients with Chronic Pancreatitis
}

\author{
Amitabh Gautam ${ }^{1}$
}

Received: 24 September 2015 / Accepted: 30 September 2015 / Published online: 2 November 2015

(C) Association of Surgeons of India 2015

The pancreas is a dual organ. The bulk (98\%) consists of the exocrine acinar tissue and the islets of Langerhans, responsible for the endocrine component constituting only about $2 \%$ of the organ. Nevertheless, loss of this endocrine component results in severe type $3 \mathrm{c}$ diabetes. The blood sugar control in these patients is very labile, perhaps because of the absence of both insulin and glucagon from loss of both beta and alpha cells of the islet of Langerhans. Chronic pancreatitis typically affects the exocrine component of the gland, and the islet structure and function is preserved unless the disease is in late advanced stages. Therefore, if total or partial pancreatectomy is indicated for pain control in chronic pancreatitis, islets can be separated from the resected pancreas, and then transplanted back into the patient, to prevent development of diabetes [1].

Paul Langerhans described the eponymous islets in the pancreas in 1869, before their function was even known. Minkowsky in 1892 first reported the development of diabetes, in dogs, after removal of the pancreas [2]. He also described that the subcutaneous placement of a portion of the pancreas prevented the mortality associated with the total pancreatectomy. Watson Williams in 1893 [2] attempted to treat diabetes, in a 15-year-old boy, by subcutaneous placement of pieces of sheep pancreas. The patient survived for a few days only, but perhaps can be considered a first attempt at a pancreatic xenograft. With the discovery of insulin from purified islet extracts, the idea of isolating islets and transplanting them

Amitabh Gautam

agautam@bu.edu

1 Section of Transplant, Department of Surgery, Boston University School of Medicine, 88 East Newton Street, D511, Boston, MA 02118, USA to reverse diabetes was being explored. Lacy and Kostianovsky, in 1967 [2], first described the technique of distending the main duct of the pancreas in rats with the collagenase enzyme, thereby digesting the exocrine tissue, and isolating purified islet suspension. The same concept was used in humans too; however, the techniques remained cumbersome, time consuming, and with poor islet yields till Camillo Ricordi reported an automated technique for islet isolation in 1986 [2]. This remains, with minor modifications, the basis for current islet extracting equipment.

The first series of human clinical islet auto transplantation, after pancreatectomy for chronic pancreatitis, was reported from the University of Minnesota in 1977. They continue to be the center with the largest experience, reporting their series of 409 patients, done up to 2011 [1]. The results of the Minnesota group are impressive. Actuarial patient survival post total pancreatectomy and islet auto transplantation was $96 \%$ in adults and $98 \%$ in children at 1 year, and 89 and $98 \%$ at 5 years. Complications requiring relaparotomy occurred in $15.9 \%$, and bleeding (9.5\%) was the most common complication. Islet function was achieved in $90 \%$ (C-peptide $0.6 \mathrm{ng} /$ $\mathrm{mL})$. At 3 years, $30 \%$ were insulin independent $(25 \%$ in adults, $55 \%$ in children) and $33 \%$ had partial function. Mean hemoglobin A1c was 7.0 in $82 \%$. Earlier pancreas surgery lowered islet yield (2712 vs 4077/ kg; $p=0.003$ ). Islet yield (2500/kg [36\%]; 2501 to 5000/kg [39\%]; 5000/kg [24\%]) correlated with degree of function with insulin-independent rates at 3 years of 12,22 , and $72 \%$, and rates of partial function 33, 62, and $24 \%$. All patients had pain before pancreatectomy and nearly all were on daily narcotics. After pancreatectomy and islet auto transplantation, $85 \%$ had pain improvement. By 2 years, $59 \%$ had ceased narcotics. All children were on narcotics before, $39 \%$ at follow-up; pain improved in $94 \%$; and $67 \%$ became pain-free. In the quality of life survey, there was significant improvement from baseline 
in all dimensions, including the physical and mental component summaries $(p<0.01)$, whether on narcotics or not.

The short-term and long-term results of auto islet transplantation are critically dependent on the number of islets infused. This in turn depends on a number of factors. First and foremost is the number of islets in the resected pancreas. More advanced the chronic pancreatitis, and therefore with more concomitant damage to the islets, gives rise to poor islet isolates and clinical results. Previous non-resectional surgery, on the diseased pancreas, also reduces the islet yield. It is important during the resection to preserve the blood supply of the pancreas till the very last stages, to prevent anoxic damage to the islets. Rapid cooling and transport of the organ after pancreatectomy, to the islet isolating facility, reduces post resectional injury to the islets. After the islets have been isolated and purified, they are suspended in solution and then infused into the portal system, by infusing through a mesenteric vein at the time of the laparotomy. This results in many cases for prolonging the anesthesia time, if the resection procedure and the biliary and enteric reconstruction are complete and the isolated islet preparation is not yet ready for transfusion. The Minnesota group has described a technique [3] of exteriorizing a small omental patch with a cannulated vein, which can be used for infusion, after the resection surgery. The infusion then takes place in the recovery area after surgery. The infused islets lodge in the portal sinusoids, were they remain engrafted. A tight glycemic control with intravenous insulin is used in the patient, in the early postoperative period, to prevent early 'stress' damage to the islets, before they recover and stabilize in their new environmental milieu. There is a small but well-described risk of portal vein thrombosis after this procedure.

From the early period, interest also remained in the use of allo islet transplants from deceased donor pancreata recovered from human organ donors. But in contrast to the auto islet transplant experience, these allo islet transplants uniformly had very short success from a few days to weeks, even with immunosuppression. The remarkably durable results reported by the Edmonton group in 2001 [4] resulted in a renewed interest and funding in islet transplantation. This has allowed development and standardization of the reagents and processes for islet isolations and purification, and increase in total islet yields from human pancreata. Best practice guidelines have appeared for islet isolation facilities, but involve a heavy financial investment in establishing and running them. Use of allo islet transplantation after pancreatectomy for chronic pancreatitis, will with the current protocols, involve giving immunosuppression with its own side effects, and is not recommended. If in the future safer tolerance protocols work [2], this may possibly become an option.

The development of a bionic pancreas [5], which uses continuous glucose monitoring, a dedicated computer program, and uses a dual insulin and glucagon pump, achieves excellent glucose control. These will be especially useful in better control of the highly labile diabetes, which results after total pancreatectomy as compared to conventional insulin pumps. These will also be useful after islet auto transplantation when there is partial response or with late failure of transplants. Practically, they are also going to be the only option, for most hospital and surgeons, who need to do the occasional resectional procedures for chronic pancreatitis and do not have access to islet isolation and auto transplantation.

\section{Compliance with Ethical Standards}

Conflict of interest No conflicts of interest.

\section{References}

1. Sutherland DE, Radosevich DM, Bellin MD, Hering BJ, Beilman GJ, Dunn TB et al (2012) Total pancreatectomy and islet autotransplantation for chronic pancreatitis. J Am Coll Surg 214: 409-424

2. Ricordi C, Strom TB (2004) Clinical islet transplantation: advances and immunological challenges. Nat Rev Immunol 4:59-268

3. Nath DS, Kellogg TA, Sutherland DE (2004) Total pancreatectomy with intraportal auto-islet transplantation using a temporarily exteriorized omental vein. J Am Coll Surg 199:994-995

4. Shapiro AJ, Lakey JR, Ryan EA, Korbutt GS, Toth E, Warnock G et al (2000) Islet transplantation in seven patients with type 1 diabetes mellitus using a glucocorticoid-free immunosuppressive regimen. $\mathrm{N}$ Engl J Med 343:230-238

5. Russell SJ, El-Khatib FH, Sinha M, Magyar KL, McKeon K, Goergen LG et al (2014) Outpatient glycemic control with a bionic pancreas in type 1 diabetes. N Engl J Med 371:313-325 